\title{
Fibroblast Growth Factor 23 Predicts All-Cause Mortality in a Dose-Response Fashion in Pre-Dialysis Patients with Chronic Kidney Disease
}

\author{
Cheng Xue ${ }^{a, c}$ Bo Yang ${ }^{a} \quad$ Chenchen Zhou $^{b}$ Bing Dai ${ }^{a} \quad Y_{\text {awei Liu }}^{a}$ Zhiguo Mao $^{a}$ \\ Shengqiang $\mathrm{Yu}^{\mathrm{a}}$ Changlin $\mathrm{Mei}^{\mathrm{a}}$ \\ ${ }^{a}$ Kidney Institute of CPLA, Division of Nephrology, Changzheng Hospital, Second Military Medical University, and \\ ${ }^{b}$ Department of Nephrology, Yueyang Hospital of Integrated Traditional Chinese and Western Medicine, Shanghai \\ University of Traditional Chinese Medicine, Shanghai, and ' Department of Pharmacy, 309 Hospital of PLA, Beijing, China
}

\section{Key Words}

Chronic kidney disease - Fibroblast growth factor 23 .

All-cause mortality · Cardiovascular diseases · End-stage renal disease $\cdot$ Meta-analysis

\begin{abstract}
Background: Quantitative dose-response associations between fibroblast growth factor 23 (FGF23) and risks of mortality, cardiovascular disease (CVD), and renal events in chronic kidney disease (CKD) are not known. This study aimed to summarize and quantify the predictive effects of FGF23 among the pre-dialysis CKD stages 1-5 population. Methods: Data sources included PubMed, EMBASE, and Web of Science. Prospective cohort studies assessing the associations between FGF23 and all-cause mortality, CVD, and renal events in CKD patients were selected. Summary risk ratios (RRs) and 95\% confidence intervals ( $\mathrm{Cls}$ ) were calculated using the random-effects model. The composite higher or the highest level in FGF23 categories of each study was considered the high level. The reference level was regarded as the low level in the overall analysis. The restricted cubic spline model was used to estimate dose-response associations. Results: Fifteen prospective cohort studies centered around 15,355 subjects were analyzed. A high FGF23 level was associated with increased risks of all-cause mortality (RR 1.46, $95 \% \mathrm{Cl} 1.38-1.55, p<0.001$ ), CVD (RR 1.37, 95\% Cl 1.15-1.63,
\end{abstract}

$p<0.001$ ), and renal events (RR 1.31,95\% Cl 1.07-1.59, $p=$ $0.008)$, respectively. There was a positive, nonlinear, doseresponse relationship between FGF23 and all-cause mortality. The reference level in dose-response analysis was defined as $51 \mathrm{RU} / \mathrm{mL}$ of c-terminal FGF23. We then calculated RRs for increments of $20 \mathrm{RU} / \mathrm{mL}$, which was associated with increased risks of mortality (RR 1.04, 95\% Cl 1.00-1.07, $p=$ $0.038), \mathrm{CVD}$ (RR 1.02, $p<0.001$ ), and renal events (RR 1.01, $p<$ $0.001)$, respectively. Conclusions: There may be positive dose-response predictive effects of FGF23 on all-cause mortality, CVD, and renal events in patients with CKD.

(c) 2017 S. Karger AG, Basel

\section{Introduction}

Chronic kidney disease (CKD) is defined as structural or functional abnormalities of the kidney for at least 3 months [1]. It is reported that $8-16 \%$ of the world's population are living with $\mathrm{CKD}$. Characterized by irreversible renal function decrease, $\mathrm{CKD}$ contributes the third largest part in years of life lost due to its unacceptable high premature mortality (82\%) [2]. The significant adverse clinical outcomes of patients with CKD include

C.X., B.Y., and C.Z. contributed equally to this work.

\section{KARGER}

(c) 2017 S. Karger AG, Basel

E-Mail karger@karger.com

www.karger.com/ajn
Changlin Mei or Bing Dai

Kidney Institute of CPLA, Division of Nephrology

Changzheng Hospital, Second Military Medical University

415 Fengyang Road, Shanghai 200003 (PR China)

E-Mail mcl312@126.com or E-Mail daibin105@126.com. 
progression to end-stage renal disease (ESRD) and development of complications (sometimes lethal). Because of the close connection between the circulation and urinary system in the water-electrolyte metabolism, the glomerular filtration rate (GFR) associated with the prevalence of cardiovascular diseases (CVDs) in the CKD population declined directly $[3,4]$. Anaemia, metabolic bone disease, and acidosis are also common complications of CKD [5-7].

It is clinically important to predict the risk of disease progression and the development of CKD complications because decisions of referral, care plans, and other therapeutic strategies, including frequency of monitoring and follow-up would be made based on the prognosis prediction. KDIGO 2012 CKD Clinical Practice Guideline suggested several factors including the degree of albuminuria, GFR, and other laboratory parameters as an indicator of identifying the CKD progression [8]. However, only ambiguous conclusions could be drawn according to these factors, since the relative weight of each of them was not clear.

Fibroblast growth factor 23 (FGF23) with endocrine effects on mineral metabolism has been in the spotlight of cardiology and nephrology research communities for decades $[9,10]$. Mounting evidence revealed the close relationship between excess serum FGF23 and cardiac events and all-cause mortality in the CKD population [11-14]. While it is not merely a biomarker, research showed the biological relevance of elevated FGF23 and adverse outcomes: apart from dysregulated phosphorus metabolism, excess FGF23 could induce vascular calcification and myocardial hypertrophy in both klotho-dependent or -independent ways $[15,16]$. Based on the associations between the FGF23 and CVD in pathogenesis, the factor is expected to be a predictor with high specificity in CVD. Besides, dose-response predictive roles of FGF23 on mortality, CVD, and renal events may exist.

The present systematic review and dose-response meta-analysis aimed at comprehensively summarizing and quantifying the predictive role of serum FGF23 for the all-cause mortality, cardiac events, renal events, and other secondary adverse clinical outcomes in the CKD stages 1-5 population.

\section{Materials and Methods}

This study was performed based on Preferred Reporting Items for Systematic Reviews and Meta-Analyses (PRISMA) criteria [17]. The registration of this review was published in PROSPERO (CRD42016041371; www.crd.york.ac.uk/PROSPERO).

\section{Literature Search}

Authors (C.X. and B.Y.) performed a literature search using the PubMed database (up to June 16, 2016), EMBASE (up to June 2016), and Web of Science database (up to June 2016). The PubMed search terms both as medical subject headings and free text terms were "FGF-23 OR FGF 23 OR fibroblast growth factor 23" and "kidney diseases" and "humans" and the search terms were adapted for the other electronic data sources. We also checked references of included articles and Google scholar for further relevant studies. There was no language restriction.

\section{Study Selection}

Both authors (C.X. and B.Y.) checked the abstracts or full texts of articles identified by the literature search independently, and screened out eligible studies. Studies were included according to the following criteria: (1) prospective cohort studies; (2) pre-dialysis patients with CKD (stages 1-5); (3) serum FGF23 level was the exposure of interest; (4) outcomes of interest were all-cause mortality or CVD (including myocardial infarction or atrial fibrillation or myocardial ischemia or coronary stenosis or stoke or aortic disease or peripheral arterial disease or heart failure or implantation of left ventricular assist device) or renal events (ESRD or initiation of dialysis or doubling of baseline serum creatinine or a decrease in GFR of $30 \%)$; (5) hazard ratio (HR) or relative risk (RR) with corresponding $95 \%$ confidence interval $(\mathrm{CI})$ were reported, or there was enough data to calculate the HR/RR. If the same study was published more than once, the publication with more detailed results was included. Studies were excluded if they were (1) reviews, case reports, abstracts, or commentaries; and (2) on pediatric and pregnant patients.

\section{Data Collection and Methodological Quality Assessment}

From each study, both authors (C.X. and B.Y.) independently extracted the following information: first author, study design, publication year, countries, study size, number of cases, gender, average age, follow-up duration, FGF23 measurement, FGF23 categories, lower and upper range of the FGF23 assay, reference levels, CKD stages, kidney functions, outcomes, and covariates adjusted for multivariable analysis.

Both authors (C.X. and B.Y.) performed the quality assessment independently. We used the Newcastle-Ottawa Scale (NOS) to evaluate methodological qualities of included studies [18]. NOS scored studies by the comparability and the selection of study groups, and the ascertainment of the interested outcome. Discrepancies were resolved by discussion and consensus. Detailed criteria of methodological quality of included studies are shown in the online supplement 1 (for all online suppl. material, see www.karger. com/doi/10.1159/000454959).

\section{Statistical Analysis}

For all-cause mortality risk, CVD risk, and renal events risk, summary HRs and 95\% CIs were calculated for a high level of FGF23 versus a low level of FGF23. Results of intact FGF23 (iFGF23) and c-terminal FGF23 (cFGF23) were calculated. The random-effects model was used in meta-analysis considering the heterogeneity from different cohort studies. HR was regarded as equivalent to $\mathrm{RR}$ in the prospective cohort study. Studies used $\mathrm{RU} / \mathrm{mL}$ as the measurement unit of cFGF23, and $\mathrm{pg} / \mathrm{mL}$ as iFGF23. The doseresponse analysis could be performed only in those studies that detected cFGF23. The reference level or the lowest level in each study was regarded as the low level in the overall analysis, while the lowest 


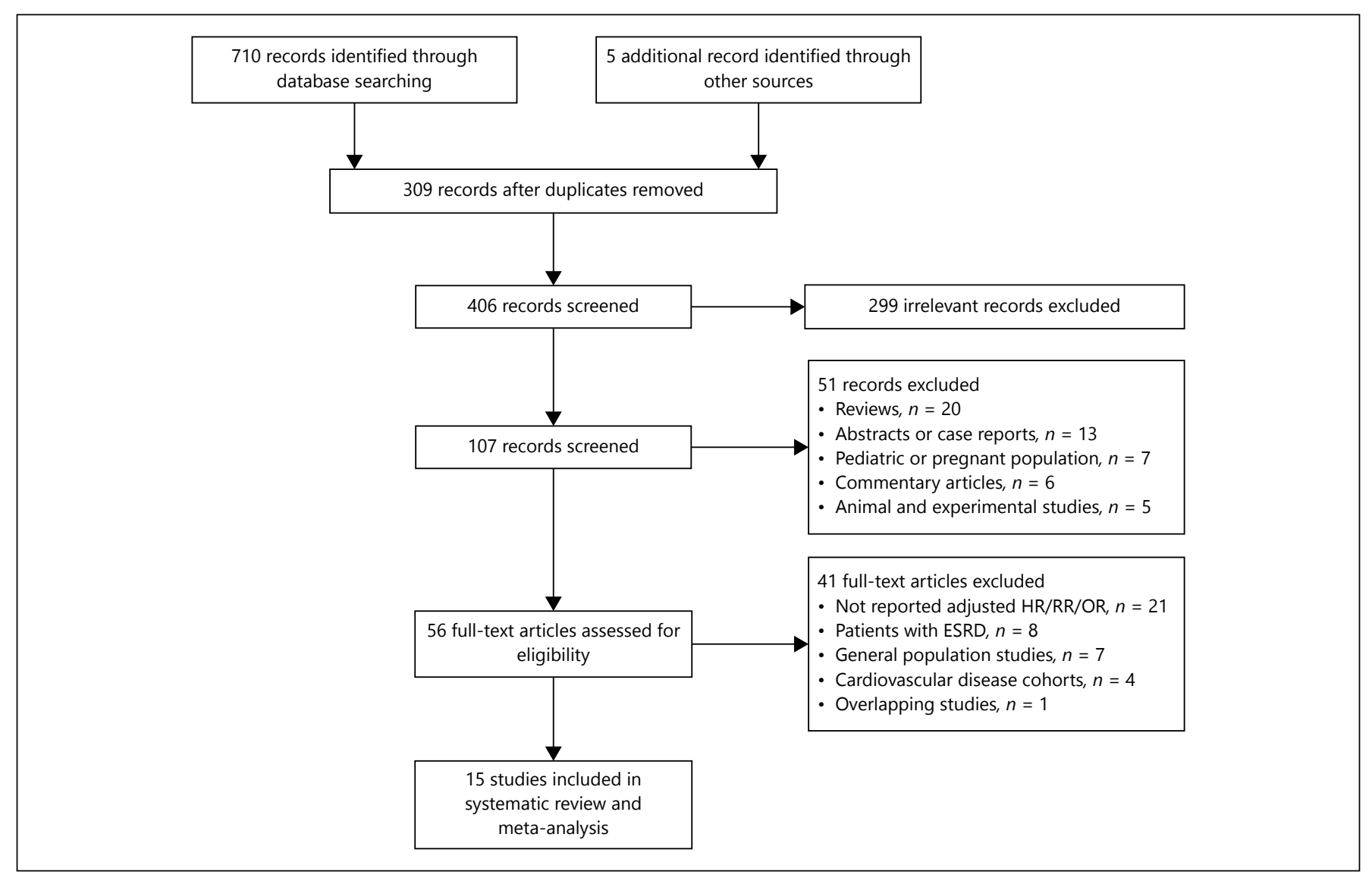

Fig. 1. Flow of study identification, inclusion, and exclusion.

reference level of $51 \mathrm{RU} / \mathrm{mL}$ was included in the studies as the low level in the dose-response analysis. When an included study compared the reference level of FGF23 with the composite higher level, we used the higher level as the high level. In studies that only compared the lowest level or the reference category of FGF23 with the higher categories ( $\geq 2$ categories), the highest category was regarded as the high level. The RRs per $20 \mathrm{RU} / \mathrm{mL}$ increase of cFGF23 level with the reference level of $51 \mathrm{RU} / \mathrm{mL}$ were calculated. We estimated the average of the natural logarithm of RRs and weighted them by the inverse of variance. Study-specific slopes, linear trends, and 95\% CIs in dose-response analysis were calculated from the natural logs of RRs and CIs across categories of FGF23 using a generalized least squares regression model [19]. The median level of FGF23, case, and person-years of each category to the corresponding RR in each study were required in the model. Relative RRs with $95 \%$ CIs for $\geq 3$ quantitative exposure categories also had to be known. The mean level between lower and upper boundaries for each FGF23 category was assigned to the relevant RR. For the open-ended lowest or highest FGF23 category, we assumed that the amplitude was equal to the adjacent category. The dose-response result in the forest plot represented a $20 \mathrm{RU} / \mathrm{mL}$ increment of cFGF23. We used nonlinear dose-response curves and the model of restricted cubic splines to check potential nonlinear relationships between the FGF23 level and corresponding risks $[19,20]$. We used 4 knots fixed at percentiles $5,35,65$, and $90 \%$ through the pooled values of FGF23 levels
[20]. We calculated the probability of nonlinearity by testing the null hypothesis that the coefficient of the second spline was zero.

Statistical heterogeneity of the results was assessed using $Q$ and $I^{2}$ statistics [21]. We considered low, moderate, and high heterogeneity to be 25,50 , and $75 \%$ of $I^{2}$ values respectively. Potential sources of heterogeneity were examined by subgroup analysis according to the ethnicity, the etiology of CKD, and the duration of follow-up. We performed meta-regression to find sources of heterogeneity. Sensitivity analysis was conducted by excluding each study at a time to find whether the result was affected by a large study or an extreme result. Funnel plots and the Begg's test were used to investigate potential small-study effects such as the publication bias [22]. All analyses were assessed using the STATA software version 12.0 (Stata, College Station, TX, USA). When $p<0.1$, the heterogeneity was significant. Otherwise, $p$ value was 2 -sided with a significance level of 0.05 .

\section{Results}

\section{Literature Search}

The process of selecting relevant studies is shown in Figure 1. The initial search found 715 studies from 
PubMed and Web of Science databases. After exclusion of irrelevant studies and duplicates, 96 potentially eligible studies were thoroughly screened. Eventually, 15 studies [23-37] were included for the final meta-analysis. The search of references from included studies did not yield additional eligible studies.

\section{Study Characteristics}

Characteristics of the included studies are listed in Table 1 . There were 15 prospective cohort studies $(n=$ 15,355) [23-37] that investigated the associations between FGF23 and CKD. Eight cohort studies $(n=$ $10,075)[26-28,30,33-36]$ investigated the association between FGF23 and all-cause mortality risk, and 8 studies $(n=8,013)[23,26-28,30,31,35,37]$ assessed the association between FGF23 and CVD risk. Eight studies $(n=9,524)[24,25,29,30,32,34-36]$ assessed the association between FGF23 and renal events risk. The duration of follow-up ranged from one year to 10.5 years [23-37]. Nine studies divided the FGF23 level into 4 categories [24, 26-28, 30-32, 34, 35]. Four studies [23, $26,29,32]$ reported iFGF23 levels, while the others reported cFGF23 levels [24, 25, 27, 28, 30, 31, 33-37]. Seven studies were performed among European populations [25, 27, 29, 32, 33, 35, 37], 3 among Asian populations [23, 24, 26], and 5 among American populations $[28,30,31,34,36]$. Methodological quality assessments are shown in the online supplement 1 , and the average quality was more than 6 [23-37]. The PRISMA checklist for this meta-analysis is listed in the online supplement 2.

\section{Quantitative Data Synthesis}

All-Cause Mortality Risk

The high level of cFGF23 was associated with an increased risk of all-cause mortality in the overall analysis using a random-effects model (7 studies [27, 28, 30, 3336], RR 1.46, 95\% CI 1.38-1.55, $p<0.001, I^{2}=0.0 \%$; Fig. 2a). There was little publication bias demonstrated by a funnel plot (Begg's test: $p=0.23$; online suppl. 3). Three studies with RR for categorized FGF23 levels were included for the dose-response analysis $[28,30,34]$. The summary RR was 1.04 (95\% CI 1.00-1.07, $p=0.038, I^{2}=$ $95.8 \%$; Fig. 2 b). There was a potentially nonlinear doseresponse relationship between FGF23 level and risk of allcause mortality ( $p<0.001$; Fig. 3 ). The association between FGF23 and the risk of all-cause mortality is depicted in the shape of a reverse J (Fig. 3). We found no significant evidence of publication bias (Begg's test: $p=$ 0.30; online suppl. 4).

\section{CVD Risk}

The high cFGF23 level was associated with an increased risk of CVD compared with the low level (6 studies $[27,28,30,31,35,37]$, RR 1.37, 95\% CI 1.15-1.63, $p<$ $0.001, I^{2}=60 \%$; Fig. $4 \mathrm{a}$ ). Publication bias was not significant by a funnel plot (Begg's test: $p=0.06$; online suppl. 7). However, the association between the iFGF23 level and the risk of CVD was not statistically significant (2 studies [23, 24], RR 1.27, 95\% CI 0.81-2.01, $p=0.298, I^{2}=$ 76.7\%; Fig. 4a). Moreover, the high cFGF23 level was associated with an increased risk of heart failure compared with the low level ( 3 studies [27, 28, 35], RR 1.54, 95\% CI $1.37-1.73, p<0.001, I^{2}=0 \%$; online suppl. 8 ). Three studies were included in the dose-response analysis $[28,30$, 31]. The summary RR for cFGF23 was 1.02 (95\% CI 1.01$1.03, p<0.001, I^{2}=0 \%$; Fig. $\left.4 \mathrm{c}\right)$. There was little evidence of publication bias by the funnel plot (Begg's test: $p=$ 0.296; online suppl. 9). There was potentially a linear dose-response relationship between FGF23 level and CVD risk ( $p=0.09$; Fig. $4 \mathrm{e})$.

\section{Renal Events Risk}

The high cFGF23 level was associated with an increased risk of renal events ( 6 studies $[25,29,30,34-36]$, RR 1.31, $95 \%$ CI 1.07-1.59, $p=0.008, I^{2}=90 \%$; Fig. 4b). The smallstudy effect was not significant (Begg's test: $p=0.260$; online suppl. 10). However, there was not a significant association between iFGF23 level and the risk of renal events (3 studies [26, 29, 32], RR 1.95, 95\% CI 0.88-4.28, $p=$ $\left.0.098, I^{2}=96.2 \%\right)$. Two studies were included in the doseresponse analysis $[30,34]$. The summary RR for per 20 units of cFGF23 was 1.010 (95\% CI 1.007-1.013, $p<0.001$; $I^{2}=0 \%$; Fig. $4 \mathrm{~d}$ ). There was little evidence of publication bias (Begg's test: $p=1.00$; online suppl. 11). A potentially linear dose-response relationship was found between cFGF23 and the risk of renal events ( $p=0.94$; Fig. $4 \mathrm{f}$ ).

Sensitivity Analysis and Subgroup Analysis

Sensitivity analysis of the overall results about cFGF23 on risks of all-cause mortality, CVD, and renal events found consistent outcomes (online suppl. 5). The positive correlations between FGF23 and the risk of all-cause mortality were similar in subgroups of the population and the follow-up duration (online suppl. 6). The correlations between FGF23 and the CVD risk were similarly significant in subgroups of American and the long follow-up duration ( $\geq 3$ years) but not in the European and the short follow-up. Results between FGF23 and the risk of renal events were significant in the subgroup of the short follow-up duration but not in different ethnicities. 


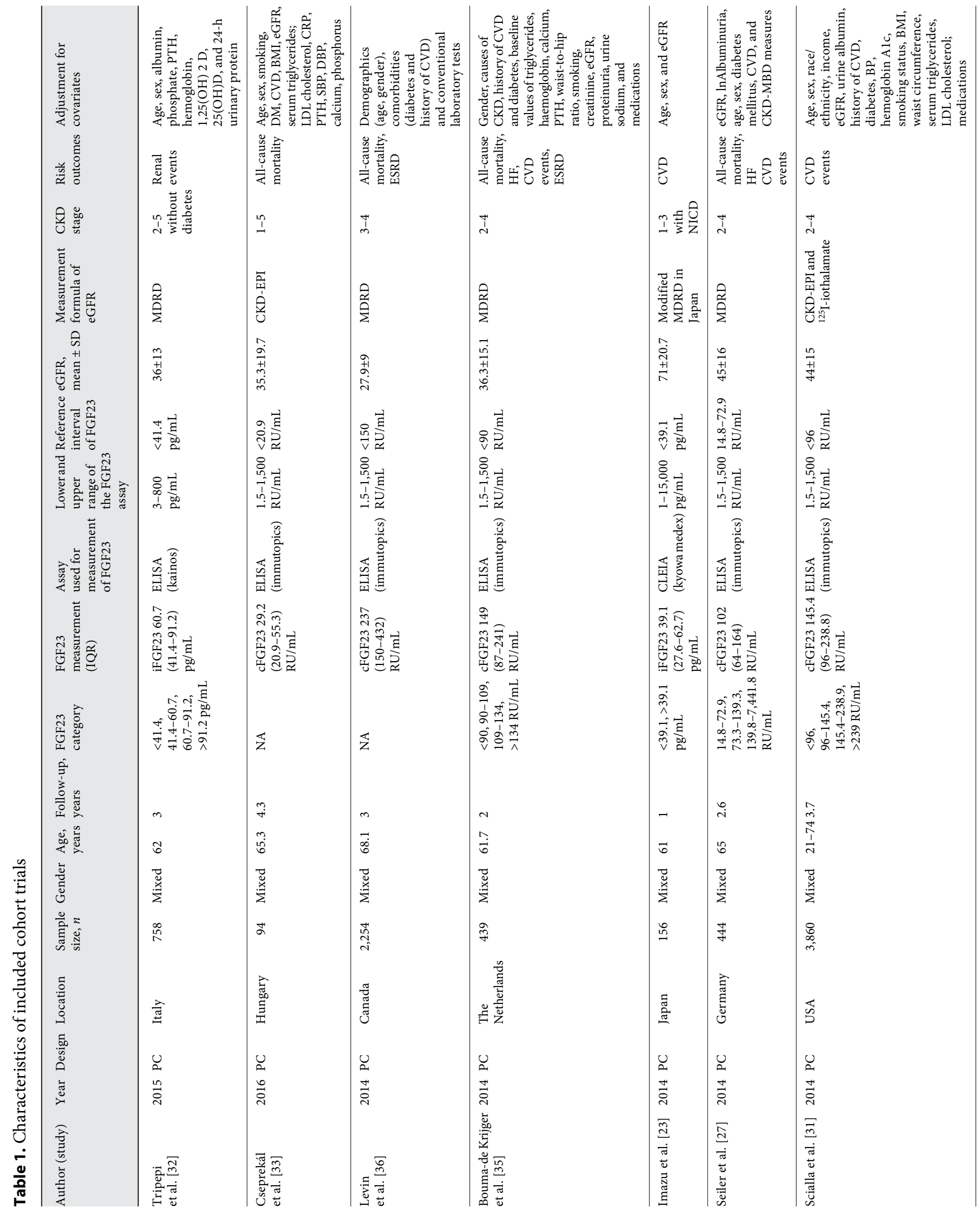




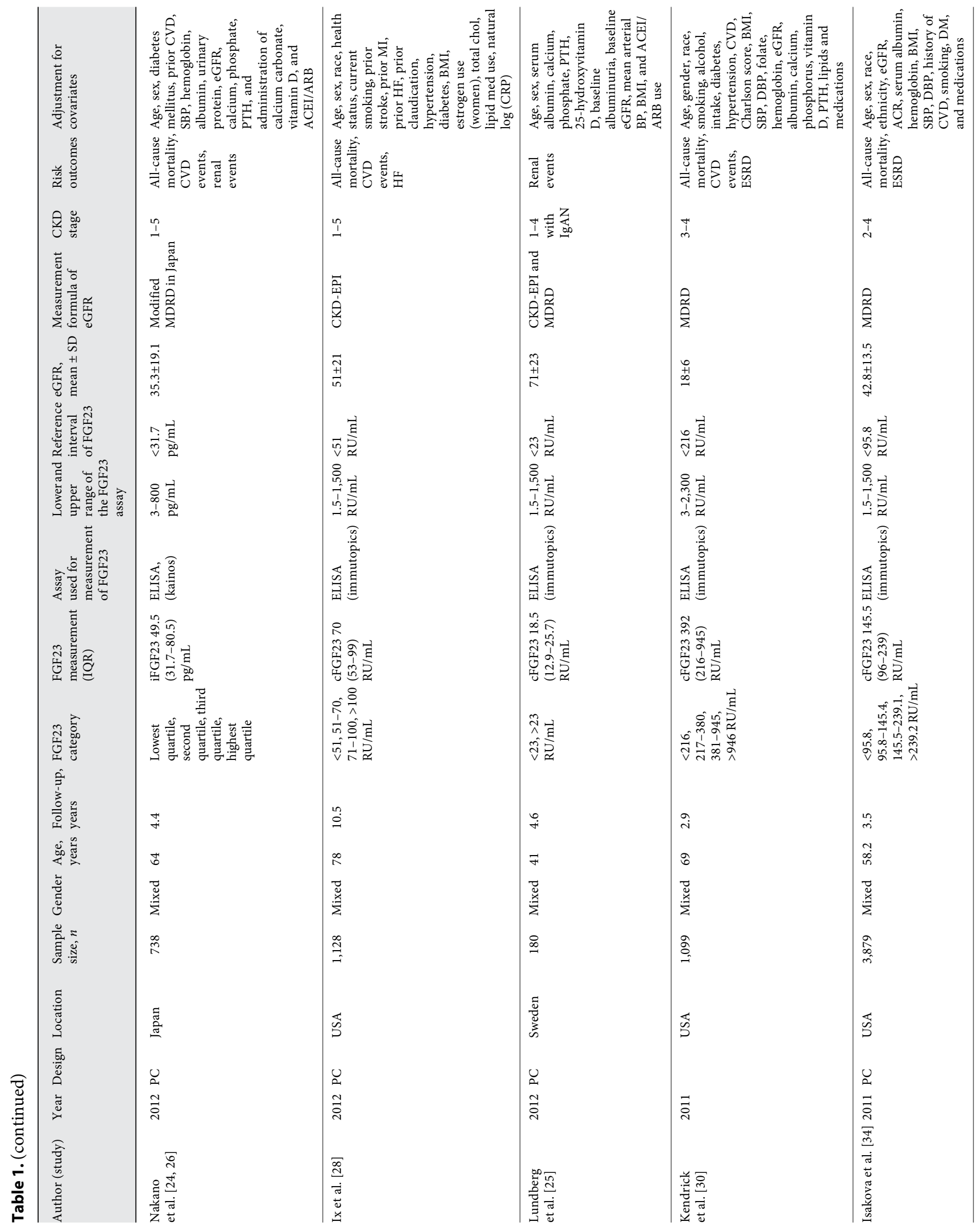




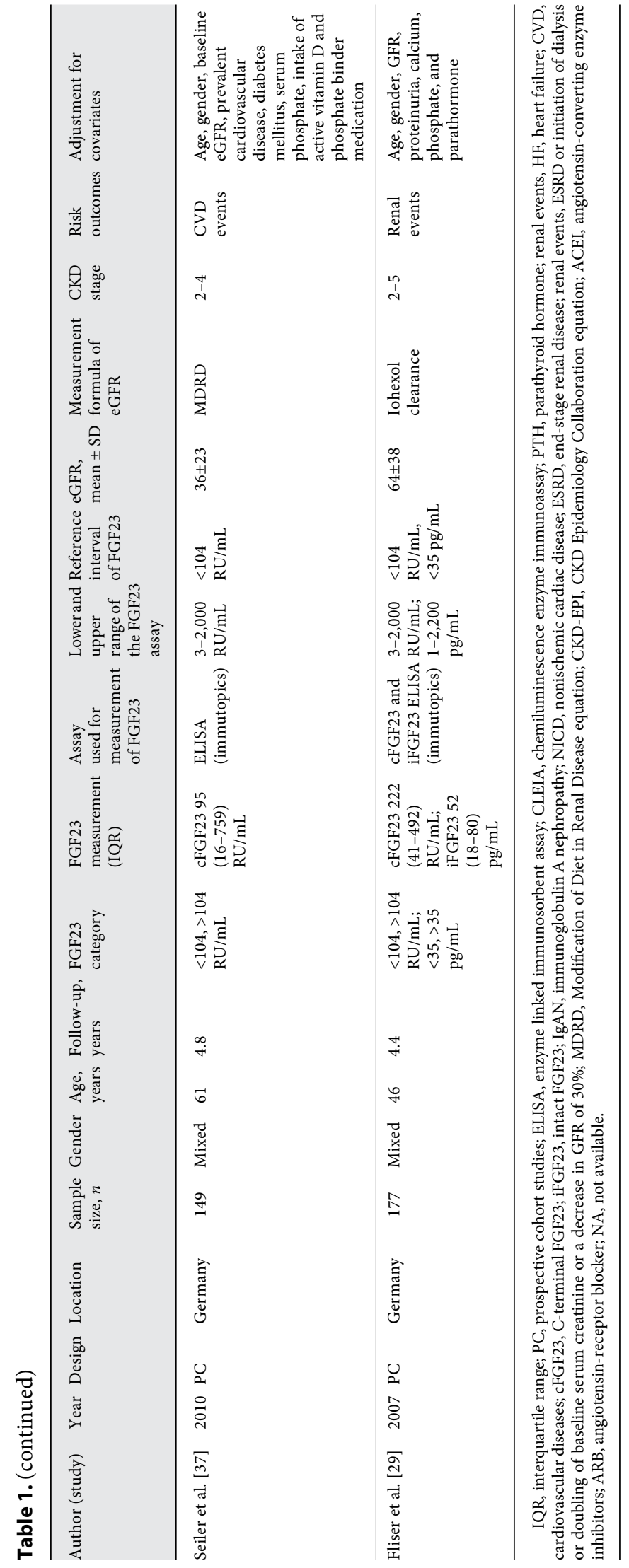

Heterogeneity Analysis

The heterogeneity mainly existed in the CVD risk and the risk of renal events. The degree of adjustment, reference levels of FGF23, and CKD stages might be the sources of heterogeneity by meta-regression. Heterogeneity of the results in CVD risk mainly came from studies conducted among the European population, and decreased $20.9 \%$ after excluding one study [23]. Heterogeneity of results about the risk of renal events mainly came from studies with the long follow-up duration.

\section{Discussion}

\section{Principal Findings and Possible Interpretations}

This systematic review and meta-analysis provided evidence for the prognosis-predictive role of serum FGF23 in pre-dialysis CKD patients. Fifteen prospective cohort studies with 15,355 subjects were analyzed. The results reveal that the high FGF23 level was associated with increased risk of renal events, CVD events, and all-cause mortality. Results of the dose-response analysis demonstrated a positive, non-linear dose-response relationship between the FGF23 level and the risk of all-cause mortality. An increment of $20 \mathrm{RU} / \mathrm{mL}$ of FGF23 concentration was associated with a $4 \%$ increased risk of mortality. Pooled analyses also indicated that in pre-dialysis CKD patients, the high level of FGF23 indicated a significantly increased risk of CVD or risk of renal events. Linear doseresponse relationships were observed between FGF23 level and risk of CVD or renal events. This systematic review included all the pre-dialysis CKD patients regardless of their original disease, which provided a good model for the whole spectrum of CKD stages $1-5$ populations. The analyses supported that FGF23 act as an independent predictor of the all-cause mortality, renal events, heart failure, and CVD in pre-dialysis CKD patients.

Results of the risks of CVD and renal events varied between the cFGF23 and the iFGF23. Different FGF23 assays could influence the results. The c-terminal enzymelinked immunosorbent assay (ELISA) could recognize cterminal-derived fragments of FGF23 proteolysis as well as iFGF23. The cFGF23 ELISA was found to be more sensitive and stable than the iFGF23 across CKD stages [38], which might restrict the use of iFGF23 in CKD. Further studies are required to validate the impact of elevated iFGF23 levels on overall mortality.

Due to the unavailability of individual information of the included subjects, it is impossible to research the interrelationship of the predictive role of FGF23 and other 


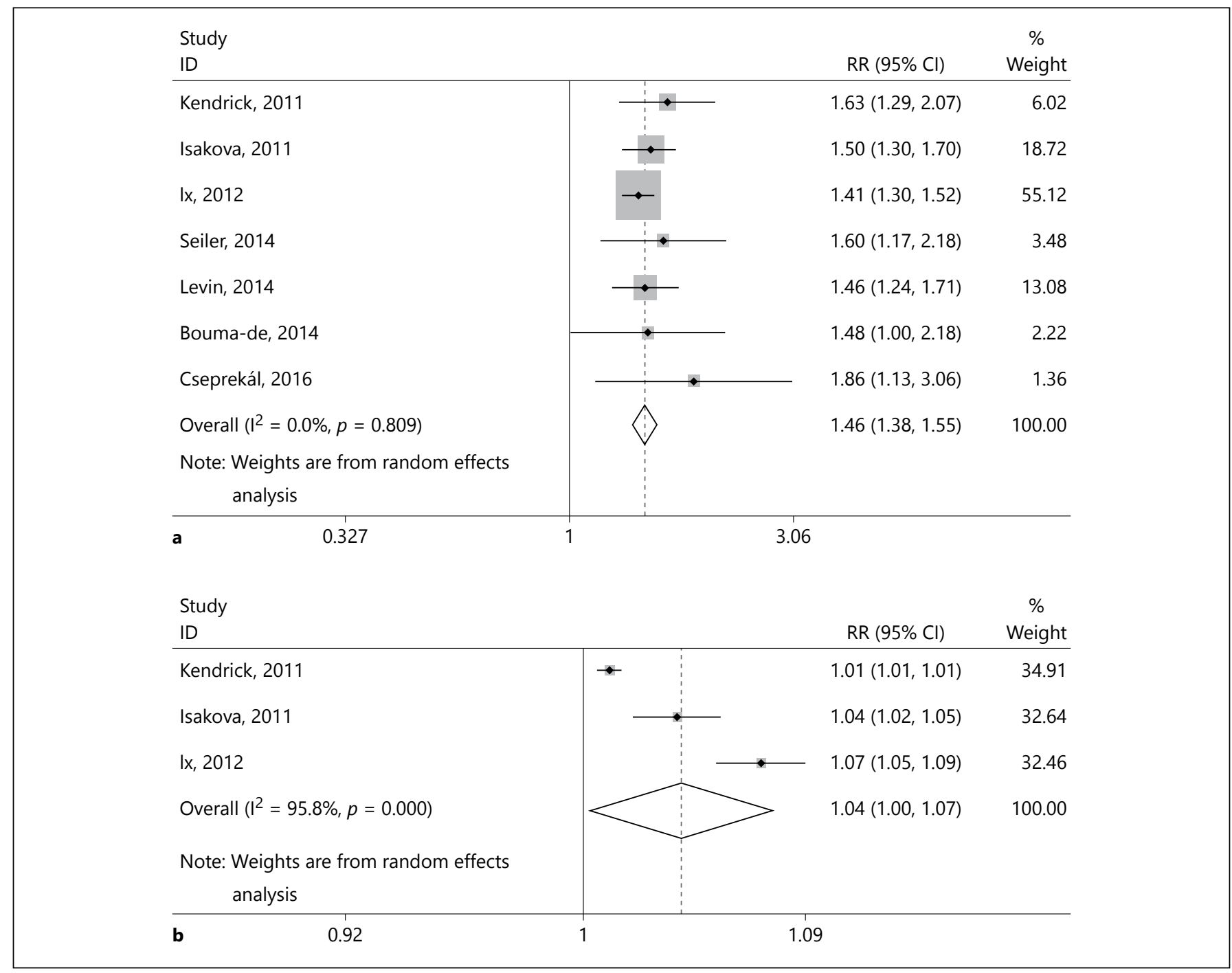

Fig. 2. Forest plots of cFGF23 level on the risk of all-cause mortality in CKD. a High vs. low analysis. b Per 20 $\mathrm{RU} / \mathrm{mL}$ increment. RR, risk ratio.

Fig. 3. The dose-response relationship between cFGF23 and the risk of all-cause mortality in CKD. FGF23 was modelled with a nonlinear trend (black continuous line) in a random effects restricted cubic spline model. Short-dashed black lines represented 95\% CIs. Long-dashed black lines represented the linear trend.

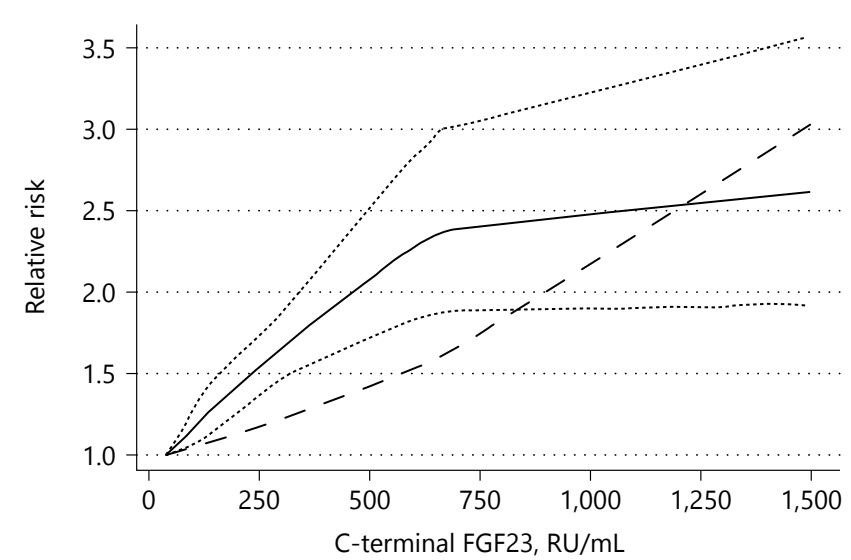

Xue/Yang/Zhou/Dai/Liu/Mao/Yu/Mei 


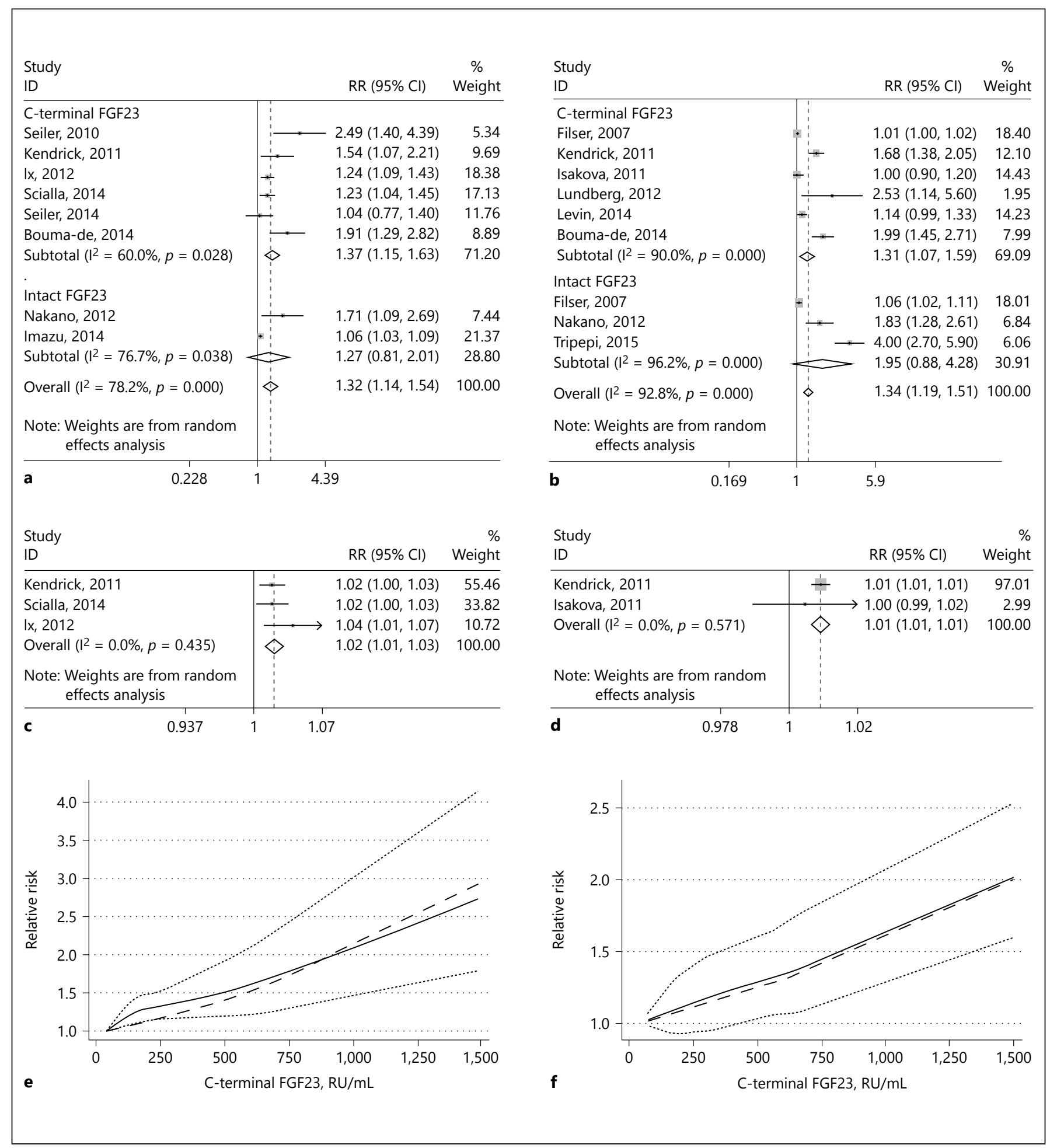

Fig. 4. Forest plots and dose-response relationships between FGF23 and risks of CVD and renal events in CKD. a High vs. low analysis of cFGF23 and iFGF23 on the risk of CVD. $\mathbf{b}$ High vs. low analysis of cFGF23 and iFGF23 on the risk of renal events. c Per 20 $\mathrm{RU} / \mathrm{mL}$ increment of the $\mathrm{CFGF} 23$ level on the risk of CVD. RR, risk ratio. d Per $20 \mathrm{RU} / \mathrm{mL}$ increment of the cFGF23 level on the risk

of renal events. e Linear dose-response relationship between cFGF23 and the risk of CVD. FGF23 was modeled with a nonlinear trend (black continuous line) in a random effects restricted cubic spline model. Short-dashed black lines represented 95\% CI. Longdashed black lines represented the linear trend. f Linear dose-response relationship between cFGF23 and the risk of renal events.

FGF23 Predicts Clinical Outcomes in 
already known predictors such as GFR and degree of albuminuria. Our results of FGF23 on risks of all-cause mortality and CKD progression provided a novel reference for the nephrologist in decision making.

\section{Comparison with Other Studies}

To our knowledge, this is the first review focusing on the predictive role of FGF23 on dialysis-independent CKD patients. The prognostic value of FGF23 in ESRD patients has been summarized previously in another meta-analysis [39]. The authors included 7 studies with 1,406 ESRD subjects. Pooled analyses indicated a higher risk of death in elevated FGF23 level patients, while doseresponse effect was not investigated in the review. For the non-CKD population, original studies revealed relatively similar conclusions $[11,40,41]$. So far, the fact that there is a relationship between FGF23 and mortality (especially cardiac mortality) has been established, while predictive model using FGF23 has not been proposed.

\section{Strengths and Limitations}

We revealed dose-response effects for all-cause mortality and CKD progression. Moreover, this study had the following strengths: more studies with a large sample size, adjustments of potential risk factors for outcomes, and including all prospective studies with low recall bias and selection bias. High NOS scores of included studies indicated satisfying methodological qualities.

There were several limitations in this study. Different reference levels of FGF23 and different FGF23 assays were the main limitations. To establish a comprehensive prediction model for CKD prognosis and complications development, there are urgent needs for a standardized assay and a standardized reference level of FGF23 in clinical practice. Most of the studies had adjusted for potential influence factors, such as age, gender, race, eGFR, smoking, calcium-phosphorus metabolism, and history of underlying disease, but the possibility of residual fac- tors could not be ruled out, such as ventricular geometry parameters for CVD. Moreover, CVD and renal events were both mixed terms, including a wide range of relevant diseases. Several studies had different definitions of these composite outcomes, so distinctions in subtypes of CVD and renal events were limited. Last, small-study effects could exist in outcomes. Studies in non-English and nonChinese or with negative results (not published) might not have been included and relevant information might have been overlooked. Overall, the results of this study need to be interpreted with caution, if necessary, and need to be confirmed by more large-scale studies in the future.

\section{Conclusions}

In summary, the high FGF23 level was associated with increased risks of all-cause mortality, CVD, and renal events. There was a positive, non-linear dose-response relationship between FGF23 and risk of all-cause mortality. Future prospective studies with a standardized FGF23 assay and adjustments for additional covariates are needed to confirm the associations.

\section{Disclosure Statement}

All the authors declared no competing interests. All authors have completed the ICMJE uniform disclosure form for Disclosure of Potential Conflicts of Interest and none were reported.

\section{Acknowledgments}

This work was supported by the National Nature Science Fund of China (No. 81370844), Chinese Society of Nephrology (No. 13030340419), China Postdoctoral Science Fund (No. 2015M572677) and Key Projects in the National Science and Technology Pillar Program in the Twelfth Five-Year Plan Period (No. 2011BAI10B00).

\section{References}

1 Levin A, Stevens PE: Summary of KDIGO 2012 CKD guideline: behind the scenes, need for guidance, and a framework for moving forward. Kidney Int 2014;85:4961.

2 Jha V, Garcia-Garcia G, Iseki K, Li Z, Naicker S, Plattner B, Saran R, Wang AY, Yang CW: Chronic kidney disease: global dimension and perspectives. Lancet 2013;382:260272 .
3 Townsend RR, Taler SJ: Management of hypertension in chronic kidney disease. Nat Rev Nephrol 2015;11:555-563.

4 Gargiulo R, Suhail F, Lerma EV: Cardiovascular disease and chronic kidney disease. Dis Mon 2015;61:403-413.

5 Summary of recommendation statements. Kidney Int Suppl (2011) 2012;2:283-287.

6 Ketteler M, Elder GJ, Evenepoel P, Ix JH, Jamal SA, Lafage-Proust MH, Shroff R, Thadhani RI,
Tonelli MA, Kasiske BL, Wheeler DC, Leonard MB: Revisiting KDIGO clinical practice guidelineonchronickidneydisease-mineralandbone disorder: a commentary from a Kidney Disease: Improving Global Outcomes controversies conference. Kidney Int 2015;87:502-528.

7 Ortega LM, Arora S: Metabolic acidosis and progression of chronic kidney disease: incidence, pathogenesis, and therapeutic options. Nefrologia 2012;32:724-730. 
8 Summary of recommendation statements. Kidney Int Suppl (2011) 2013;3:5-14.

9 Quarles LD: Endocrine functions of bone in mineral metabolism regulation. J Clin Invest 2008;118:3820-3828.

10 Wolf M: Forging forward with 10 burning questions on FGF23 in kidney disease. J Am Soc Nephrol 2010;21:1427-1435.

11 Seiler S, Cremers B, Rebling NM, Hornof F, Jeken J, Kersting S, Steimle C, Ege P, Fehrenz M, Rogacev KS, Scheller B, Bohm M, Fliser D, Heine GH: The phosphatonin fibroblast growth factor 23 links calcium-phosphate metabolism with left-ventricular dysfunction and atrial fibrillation. Eur Heart J 2011;32: 2688-2696.

12 Wolf M: Update on fibroblast growth factor 23 in chronic kidney disease. Kidney Int 2012; 82:737-747.

13 Isakova T, Ix JH, Sprague SM, Raphael KL, Fried L, Gassman JJ, Raj D, Cheung AK, Kusek JW, Flessner MF, Wolf M, Block GA: Rationale and approaches to phosphate and fibroblast growth factor 23 reduction in CKD. J Am Soc Nephrol 2015;26:2328-2339.

14 Smith ER: The use of fibroblast growth factor 23 testing in patients with kidney disease. Clin J Am Soc Nephrol 2014;9:1283-1303.

15 Scialla JJ: Epidemiologic insights on the role of fibroblast growth factor 23 in cardiovascular disease. Curr Opin Nephrol Hypertens 2015;24:260-267.

16 Tsuchiya K, Nagano N, Nitta K: Klotho/ FGF23 axis in CKD. Contrib Nephrol 2015; 185:56-65.

17 Stewart LA, Clarke M, Rovers M, Riley RD, Simmonds M, Stewart G, Tierney JF: Preferred reporting items for systematic review and meta-analyses of individual participant data: the PRISMA-IPD statement. JAMA 2015;313: 1657-1665.

18 Stang A: Critical evaluation of the NewcastleOttawa scale for the assessment of the quality of nonrandomized studies in meta-analyses. Eur J Epidemiol 2010;25:603-605.

19 Greenland S, Longnecker MP: Methods for trend estimation from summarized dose-response data, with applications to meta-analysis. Am J Epidemiol 1992;135:1301-1309.

20 Orsini N, Li R, Wolk A, Khudyakov P, Spiegelman D: Meta-analysis for linear and nonlinear dose-response relations: examples, an evaluation of approximations, and software. Am J Epidemiol 2012;175:66-73.

21 Higgins JP, Thompson SG, Deeks JJ, Altman DG: Measuring inconsistency in meta-analyses. BMJ 2003;327:557-560.

22 Egger M, Davey Smith G, Schneider M, Minder C: Bias in meta-analysis detected by a simple, graphical test. BMJ 1997;315:629-634.

23 Imazu M, Takahama $\mathrm{H}$, Asanuma $\mathrm{H}$, Funada A, Sugano Y, Ohara T, Hasegawa T, Asakura
M, Kanzaki H, Anzai T, Kitakaze M: Pathophysiological impact of serum fibroblast growth factor 23 in patients with nonischemic cardiac disease and early chronic kidney disease. Am J Physiol Heart Circ Physiol 2014; 307:H1504-H1511.

24 Nakano C, Hamano T, Fujii N, Obi Y, Matsui I, Tomida K, Mikami S, Inoue K, Shimomura A, Nagasawa Y, Okada N, Tsubakihara Y, Rakugi H, Isaka Y: Intact fibroblast growth factor 23 levels predict incident cardiovascular event before but not after the start of dialysis. Bone 2012;50:1266-1274.

25 Lundberg S, Qureshi AR, Olivecrona S, Gunnarsson I, Jacobson SH, Larsson TE: FGF23, albuminuria, and disease progression in patients with chronic IgA nephropathy. Clin J Am Soc Nephrol 2012;7:727-734.

26 Nakano C, Hamano T, Fujii N, Matsui I, Tomida K, Mikami S, Inoue K, Obi Y, Okada N, Tsubakihara Y, Isaka Y, Rakugi H: Combined use of vitamin D status and FGF23 for risk stratification of renal outcome. Clin J Am Soc Nephrol 2012;7:810-819.

27 Seiler S, Rogacev KS, Roth HJ, Shafein P, Emrich I, Neuhaus S, Floege J, Fliser D, Heine GH: Associations of FGF-23 and sKlotho with cardiovascular outcomes among patients with CKD stages 2-4. Clin J Am Soc Nephrol 2014;9:1049-1058.

28 Ix JH, Katz R, Kestenbaum BR, de Boer IH, Chonchol M, Mukamal KJ, Rifkin D, Siscovick DS, Sarnak MJ, Shlipak MG: Fibroblast growth factor-23 and death, heart failure, and cardiovascular events in community-living individuals: CHS (cardiovascular health study). J Am Coll Cardiol 2012;60:200-207.

29 Fliser D, Kollerits B, Neyer U, Ankerst DP, Lhotta K, Lingenhel A, Ritz E, Kronenberg F, Kuen E, Konig P, Kraatz G, Mann JF, Muller GA, Kohler H, Riegler P: Fibroblast growth factor 23 (FGF23) predicts progression of chronic kidney disease: the Mild to Moderate Kidney Disease (MMKD) study. J Am Soc Nephrol 2007;18:2600-2608.

30 Kendrick J, Cheung AK, Kaufman JS, Greene T, Roberts WL, Smits G, Chonchol M: FGF-23 associates with death, cardiovascular events, and initiation of chronic dialysis. J Am Soc Nephrol 2011;22:1913-1922.

31 Scialla JJ, Xie H, Rahman M, Anderson AH, Isakova T, Ojo A, Zhang X, Nessel L, Hamano T, Grunwald JE, Raj DS, Yang W, He J, Lash JP, Go AS, Kusek JW, Feldman H, Wolf M: Fibroblast growth factor-23 and cardiovascular events in CKD. J Am Soc Nephrol 2014;25: 349-360.

32 Tripepi G, Kollerits B, Leonardis D, Yilmaz MI, Postorino M, Fliser D, Mallamaci F, Kronenberg F, Zoccali C: Competitive interaction between fibroblast growth factor 23 and asymmetric dimethylarginine in patients with
CKD. J Am Soc Nephrol 2015;26:935-944.

33 Cseprekal O, Egresits J, Tabak A, Nemcsik J, Jarai Z, Babos L, Fodor E, Farkas K, Godina G, Karpathi KI, Kerkovits L, Marton A, Nemcsik-Bencze Z, Nemeth Z, Sallai L, Kiss I, Tisler A: The significance of micro- and macrovascular biomarkers on cardiovascular outcome in chronic kidney disease: a prospective cohort study. J Hum Hypertens 2016;30:449455.

34 Isakova $\mathrm{T}$, Xie $\mathrm{H}$, Yang $\mathrm{W}$, Xie D, Anderson AH, Scialla J, Wahl P, Gutierrez OM, Steigerwalt S, He J, Schwartz S, Lo J, Ojo A, Sondheimer J, Hsu CY, Lash J, Leonard M, Kusek JW, Feldman HI, Wolf M: Fibroblast growth factor 23 and risks of mortality and end-stage renal disease in patients with chronic kidney disease. JAMA 2011;305:2432-2439.

35 Bouma-de Krijger A, Bots ML, Vervloet MG, Blankestijn PJ, Ter Wee PW, van Zuilen AD, Wetzels JF: Time-averaged level of fibroblast growth factor-23 and clinical events in chronic kidney disease. Nephrol Dial Transplant 2014;29:88-97.

36 Levin A, Rigatto C, Barrett B, Madore F, Muirhead N, Holmes D, Clase CM, Tang M, Djurdjev O: Biomarkers of inflammation, fibrosis, cardiac stretch and injury predict death but not renal replacement therapy at 1 year in a Canadian chronic kidney disease cohort. Nephrol Dial Transplant 2014;29:10371047.

37 Seiler S, Reichart B, Roth D, Seibert E, Fliser D, Heine GH: FGF-23 and future cardiovascular events in patients with chronic kidney disease before initiation of dialysis treatment. Nephrol Dial Transplant 2010;25:39833989.

38 Bozentowicz-Wikarek M, Owczarek A, Kocelak P, Olszanecka-Glinianowicz M, Wiecek A, Chudek J: C-terminal to intact fibroblast growth factor 23 ratio in relation to estimated glomerular filtration rate in elderly population. Kidney Blood Press Res 2016;41:519526.

39 Yang H, Luo H, Tang X, Zeng X, Yu Y, Ma L, Fu P: Prognostic value of FGF23 among patients with end-stage renal disease: a systematic review and meta-analysis. Biomark Med 2016; 10:547-556.

40 Parker BD, Schurgers LJ, Brandenburg VM, Christenson RH, Vermeer C, Ketteler M, Shlipak MG, Whooley MA, Ix JH: The associations of fibroblast growth factor 23 and uncarboxylated matrix Gla protein with mortality in coronary artery disease: the Heart and Soul Study. Ann Intern Med 2010;152:640-648.

41 Mirza MA, Larsson A, Melhus H, Lind L, Larsson TE: Serum intact FGF23 associate with left ventricular mass, hypertrophy and geometry in an elderly population. Atherosclerosis 2009;207:546-551. 